Félicie Pastoré, Compréhension de l'oral et prises de notes : cours, conférences, congrès. Anglais professionnel pour les sciences de la santé

Paris : Ellipses, 2013

\title{
Pascaline Faure
}

\section{OpenEdition}

\section{Journals}

Édition électronique

URL : http://journals.openedition.org/asp/4556

DOI : 10.4000/asp.4556

ISSN : 2108-6354

\section{Éditeur}

Groupe d'étude et de recherche en anglais de spécialité

\section{Édition imprimée}

Date de publication : 1 novembre 2014

Pagination : 169-170

ISSN : 1246-8185

Référence électronique

Pascaline Faure, «Félicie Pastoré, Compréhension de l'oral et prises de notes : cours, conférences,

congrès. Anglais professionnel pour les sciences de la santé », ASp [En ligne], 66 | 2014, mis en ligne le 27 octobre 2014, consulté le 06 novembre 2020. URL : http://journals.openedition.org/asp/4556 ; DOI : https://doi.org/10.4000/asp.4556

Ce document a été généré automatiquement le 6 novembre 2020.

Tous droits réservés 


\section{Félicie Pastoré, Compréhension de} l'oral et prises de notes : cours, conférences, congrès. Anglais professionnel pour les sciences de la santé

Paris : Ellipses, 2013

Pascaline Faure

\section{RÉFÉRENCE}

Pastoré, Félicie. 2013. Hear that? Compréhension de l'oral et prises de notes : cours, conférences, congrès, Anglais professionnel pour les sciences de la santé. Paris : Ellipses, 216 p. ISBN : 978-2-729-88245-7. 
$1 \quad$ Hear that? Compréhension de l'oral et prises de notes: cours, conférences, congrès, Anglais professionnel pour les sciences de la santé est un recueil d'exercices axés sur la compréhension orale destiné aux professionnels de santé pour un travail en autonomie. Cette compilation d'activités audio-orales proposées à la Faculté de médecine Toulouse-Purpan est organisée autour de six thématiques (Medical education, Public health, Influenza, International travel, Tropical medicine, HIV/ AIDS). Chaque unité se décompose en trois sous-parties : Ready présente les concepts clés ainsi que des liens vers des sites de référence; Steady permet l'introduction $\mathrm{du}$ vocabulaire (avec un lien vers un fichier sonore); Go! propose des liens vers des vidéos et podcasts de longueur et de

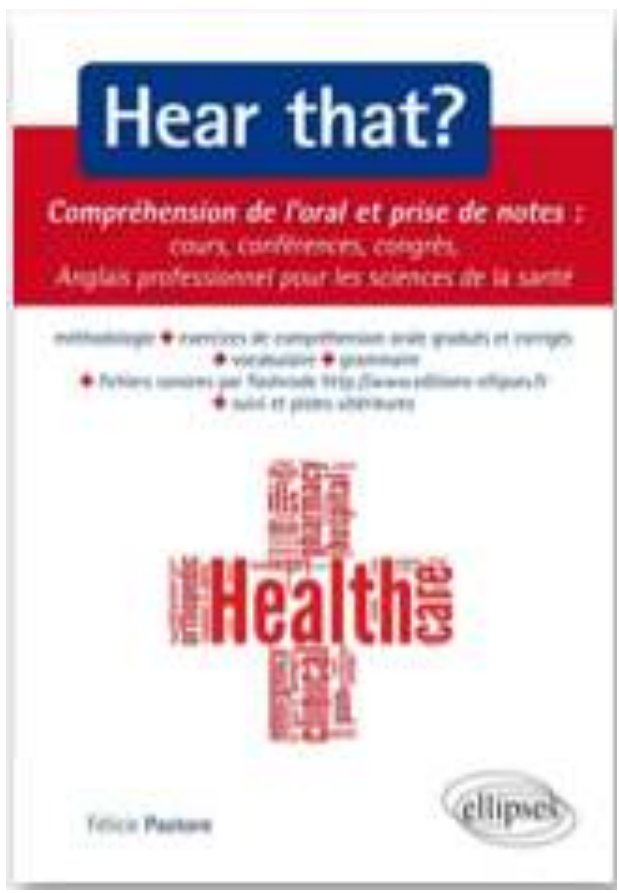
difficulté croissantes avec, pour chaque document, une fiche de compréhension qui permet de guider l'écoute; une liste de mots et d'expressions explicités (extraits du document) ; une section Language corner, qui comprend quelques remarques d'ordre plus général portant sur la grammaire par exemple et un exercice (généralement des phrases de thème) dont le corrigé se trouve à la fin du recueil ; une partie conclusive intitulée Follow-up, qui propose des tâches de type "faire un résumé ", "rédiger un essay" ou encore "présenter l'article à un collègue à l'oral en deux minutes »; et enfin le corrigé de la fiche de compréhension. La plupart des documents sont issus du site Medscape, accessible gratuitement après enregistrement.

2 La fiche de compréhension est constituée d'intitulés plus que de questions. Peut-être aurait-il fallu, pour des apprenants dont le niveau nécessite un guidage plus explicite, des questions rédigées? Nous pouvons également nous interroger sur l'utilité des key terms de la section Ready, qui paraissent un peu trop vagues. En revanche, la fiche (section Steady), qui reprend les mots-clés du document avec leur traduction et un lien vers leur prononciation, apparaît fondamentale. En outre, la présence systématique du code (ex. U5-0) après chaque mot rend la lecture laborieuse. Une transcription phonétique aurait été plus opportune. Si les faits grammaticaux sont généralement correctement présentés, certaines explications telles que "présent + aspect (be+ING) pour un événement en cours» (p.147) ou l'association «futur/will» (p.121) sont gênantes. Par ailleurs, il n'a pas toujours été facile d'accéder à tous les documents. Il est souvent nécessaire de taper le titre entier pour les trouver. L'insertion d'un index ne semble pas utile. La présentation générale est claire, mais aurait mérité quelques couleurs afin de rendre l'ouvrage visuellement plus attractif.

3 Ce recueil devrait cependant se révéler très utile aux apprenants en autonomie car le guidage est intelligemment construit et les documents soigneusement sélectionnés. Il devrait également intéresser les enseignants d'anglais médical car il s'agit d'une compilation précieuse de cours de compréhension orale «clés en main ». 


\section{AUTEURS}

PASCALINE FAURE

Université Pierre et Marie Curie, Paris 6. pascalinefaure@orange.fr 